\title{
Avaliação do sistema Quantec SC usado nas velocidades de 350 ou 700rpm em retratamento endodôntico
}

\author{
Evaluation of Quantec SC instrument, using speed at 350 or 700rpm in \\ endodontic retreatment
}

\author{
Clóvis Monteiro BRAMANTE \\ Ivaldo Gomes de MORAES \\ Norberti BERNARDINELI \\ Roberto Brandão GARCIA
}

Professor de Endodontia - Faculdade de Odontologia de Bauru - Universidade de São Paulo - USP - Bauru - SP - Brasil.

\section{Sergio Arturo SOTO CASTILLO}

Mestre de Endodontia - Faculdade de Odontologia de Bauru - Universidade de São Paulo - USP - Bauru - SP - Brasil.

\author{
Alexandre Silva BRAMANTE \\ Doutor em Endodontia Faculdade de Odontologia de Bauru - Universidade de São Paulo - USP - Bauru - SP - Brasil.
}

\begin{abstract}
Resumo
O objetivo deste estudo foi analisar a capacidade do sistema rotatório Quantec SC de remover guta-percha e/ou cimento de canais quando usado na velocidade de 350 ou 700rpm. Vinte incisivos inferiores extraídos foram obturados pela técnica da condensação lateral com guta-percha e cimento Ketac-Endo. Após seis meses as obturações foram removidas com o sistema rotatório Quantec SC na velocidade de 350 ou 700rpm. Inicialmente foram avaliados o tempo para a remoção da obturação, a extrusão de material obturador bem como o desvio do trajeto do canal, perfuração e a fratura de instrumento. A seguir os dentes foram seccionados longitudinalmente e a limpeza das paredes dos canais foi avaliada usando o software Sigma Scan. Aos dados obtidos foi aplicado o teste estatístico de Kruskal-Wallis. Observou-se que todos os canais exibiram resíduos de material obturador; a 700rpm o tempo de remoção da obturação foi menor, porém, a 350rpm menor quantidade de resíduos de guta-percha e cimento foram observadas. Quanto a extrusão de material obturador, não foram observadas diferenças estatísticas entre as velocidades de 350 e $700 \mathrm{rpm}$. Fraturas de instrumento ocorreram em ambas as velocidades sendo mais freqüentes no instrumento $\mathrm{n}^{\circ} 5$; o número de perfurações e desvios do trajeto do canal foi maior na velocidade de 350rpm. Conclui-se que os instrumentos rotatórios do sistema Quantec SC podem ser utilizados para desobstrução de canais na velocidade de 700rpm.
\end{abstract}

\section{UNITERMOS}

Retratamento; retratamento de canais radiculares; Quantec.

\section{INTRODUÇÃO}

O tratamento endodôntico, nos dias atuais, permite um índice de sucesso ao redor de $90 \%$. Todavia, por motivos anatômicos e técnicos, pode ocorrer um fracasso, havendo a necessidade de retratamento. Este requer uma adequada avaliação das condições do tratamento atual e, decidido pelo retratamento, a completa remoção do material obturador, nova instrumentação, desinfecção e obturação do sistema de canais radiculares $\mathbf{s}^{1,7,14,15,17,18}$.
Diferentes técnicas têm sido usadas para a remoção do material obturador, incluindo instrumentos manuais, rotatórios, oscilatórios ultra-som, com ou sem o uso de solventes ${ }^{2,3,4,10,16,20,21}$. As técnicas automatizadas têm sido propostas como alternativa à instrumentação manual, no retratamento, para remoção do material obturador 8,12,13,16,19,20,21,25,26. Diversos sistemas foram introduzidos no mercado endodôntico, destacando-se entre eles o Profile, K3, Pro tapper, Race, Hero 642 e o Quantec. Os instrumentos do sistema Quantec são feitos de níquel-titânio, compõe-se de 10 instrumentos 
numerados de 1 a 10, assim distribuídos: 1-25/06, 2-15/02, 3-20/02, 4-25/02, 5-25/03, 6-24/04, 7-25/05, 8-25/06, 9-40/02 e 10-45/02. Esses instrumentos são utilizados na velocidade de $300 \mathrm{rpm}$, porém não se encontra na literatura informação da velocidade a ser empregada quando eles são utilizados para o retratamento $^{4,18,20,21}$

O objetivo deste estudo foi analisar a capacidade dos instrumentos do sistema Quantec SC (Sybron, Orange, CA, USA) na remoção do material obturador do canal radicular quando empregados na velocidade de 350 ou 700rpm.

\section{Material e Métodos}

Vinte incisivos inferiores foram selecionados, por meio de radiografia, de modo que apresentassem raiz completamente formada, canal único e nenhuma obstrução do mesmo. Após a abertura coronária, um instrumento foi inserido no canal até que sua ponta fosse visualizada no forame oferecendo assim o comprimento do dente. Dessa medida diminui-se um milímetro estabelecendo-se a extensão de trabalho. Os canais foram instrumentados até a lima tipo $\mathrm{K} \mathrm{n}^{\circ} 40$ (Maillefer, Dentsply, Ballaigues, Switzerland) e então obturados pela técnica da condensação lateral com cimento Ketac-Endo (Ketac Endo, Espe Germany) e guta-percha (Maillefer, Dentsply, Ballaigues, Switzerland). $\mathrm{O}$ acesso endodôntico foi selado com IRM (Dentsply, Petrópolis, RJ, Brasil) e os dentes foram armazenados em $100 \%$ de umidade a $37^{\circ} \mathrm{C}$ durante seis meses.

Após esse período, os dentes foram distribuídos aleatoriamente em dois grupos (I e II) de 10 espécimes cada um. O IRM foi removido e a remoção do material obturador foi feita com os instrumentos rotatórios Quantec SC (Sybron Dental, Orange, CA, USA) trabalhando na extensão de instrumentação e acionados com motor TC 3000 (Nouvag G, Goldach, Switzerland). No grupo I, foi usada a velocidade de $350 \mathrm{rpm}$ e no grupo II a de 700rpm. A remoção era considerada completada quando não se observava mais material obturador saindo do canal radicular. Cada instrumento foi utilizado em cinco retratamentos. Após a remoção realizou-se a irrigação com $6 \mathrm{~cm}^{3}$ de hipoclorito de sódio a $1 \%$, para cada dente.

O tempo requerido para a desobturação foi medido da seguinte forma: Tempo A (Tempo inicial-tempo requerido para atingir a extensão de trabalho) desde $\mathrm{o}$ início do procedimento com o instrumento $\mathrm{n}^{\mathrm{0}} 1$ até atingir o comprimento desejado com o instrumento $\mathrm{n}^{\mathrm{0}} 5$ ; Tempo B (tempo para remover a obturação), desde o momento do uso do instrumento $\mathrm{n}^{\circ} 6$ até o final do procedimento usando o instrumento ${ }^{\circ} 10$ (limpeza do terço cervical, médio e apical). O Tempo Total foi a soma dos tempos A e B.

A quantidade de material extruído também foi avaliada. Para isso, o ápice do dente foi incluído em um alvéolo artificial confeccionado de silicone (Figura 1). Esse alvéolo foi pesado antes e depois do procedimento de desobturação.
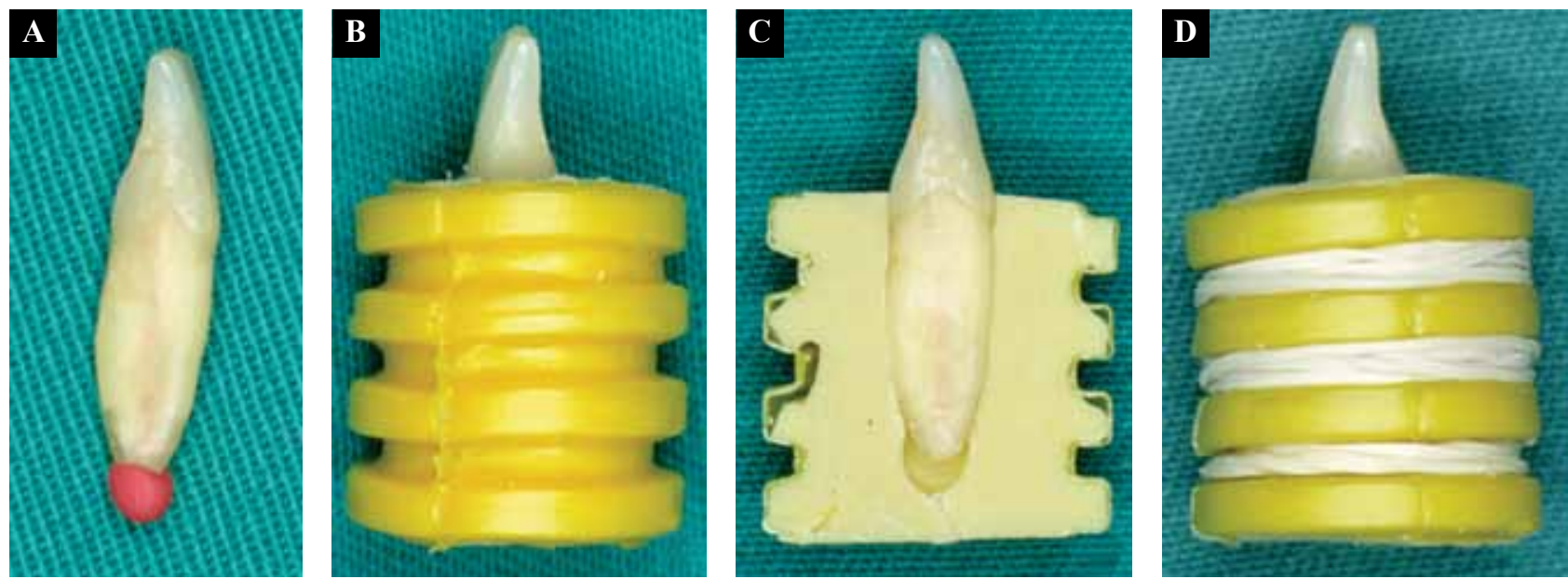

Figura 1 - Confecção do alvéolo artificial para avaliação da quantidade de material extruído: a-dente com cera no ápice; b-dente incluído em silicone; c-hemiparte do conjunto mostrando espaço no ápice para coleta do material extruído; d-conjunto montado. 
Número de desvio do trajeto do canal, perfuração e fratura de instrumento foram avaliados macroscópica e radiograficamente.

Nas faces vestibular e lingual dos dentes foram confeccionados sulcos longitudinais com disco diamantado em baixa velocidade até as proximidades do canal e, então, os dentes foram seccionados com cinzel. Cada metade foi escaneada com o Scanjet II 4C (Hewlett Packard, Palo Alto, CA, USA), com resolução de 300dpi, e avaliada usando o software Sigma Scan II (Jandel Scientific, Chicago, USA); as imagens foram ampliadas 4 vezes de seu tamanho original. Os canais foram divididos em terços e cada um foi avaliado em separado. As áreas ocupadas pelo material obturador (guta-percha e cimento) que permaneceu na parede dos canais foram quantificadas em $\mathrm{mm}^{2}$ calculando-se a porcentagem ocupada por esses materiais em relação às áreas correspondentes aos terços (Figura 2).

A análise de variância a um critério pelo teste de Kruskal-Wallis foi usada visando identificar possíveis diferenças entre os dois grupos. Quando encontrada, o teste $\mathrm{T}$ foi aplicado para determinar essa diferença.

\section{Resultados}

A Tabela 1 mostra os tempos médios (A,B e total) para a remoção do material obturador, a quantidade (\%) de material remanescente nas paredes do canal e a extrusão de material (em gramas) após o procedimento de desobturação com o instrumento rotatório
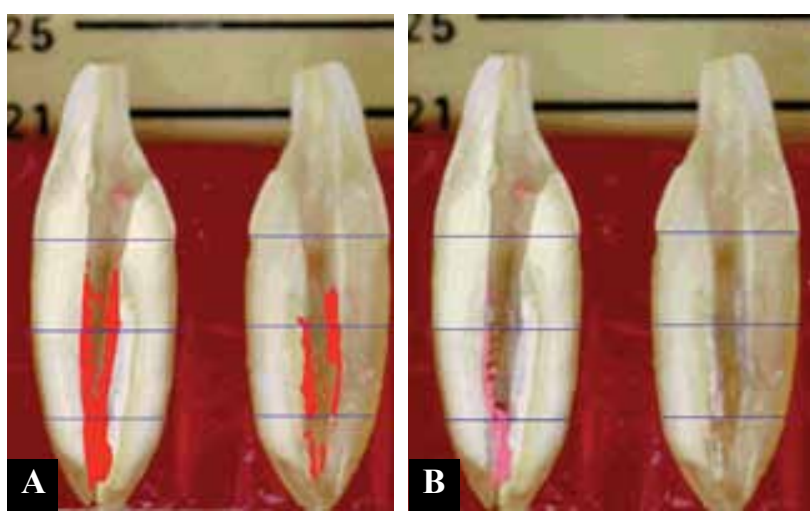

Figura 2 - Avaliação dos resíduos de material obturador nos terços cervical, médio e apical: a-demarcação da área contendo o resíduo de material obturador; b-raiz dividida em terços, cervical, médio e apical mostrando resíduo de guta-percha e cimento.

Quantec SC na velocidade de 350 ou 700rpm. No teste $\mathrm{T}$, foi constatada diferença entre o tempo total das duas velocidades $(p<0.05)$. Essa diferença também foi evidente entre os tempos A e B $(p<0.05)$; o teste $T$ mostrou diferença entre as velocidades $(p<0.05)$ na remoção do material obturador com vantagem para a velocidade de 350rpm. Nos terços cervical e médio houve diferença estatística entre as duas velocidades utilizadas $(\mathrm{p}<0.05)$. Não foi constatada diferença (teste $\mathrm{T}$ ) com relação à extrusão de material. Na Tabela 2 esta o número de acidentes (fraturas ocorridas com os diferentes instrumentos, desvios e perfurações do trajeto do canal).

Tabela 1 - Tempos médios (minutos), resíduo de material (\%) e extrusão (g) de material obturador em função da velocidade (350 ou 700rpm), durante a desobturação dos canais radiculares

\begin{tabular}{|c|c|c|c|c|c|c|c|}
\hline \multirow{3}{*}{$\begin{array}{l}\text { VELOCIDADE } \\
\text { (rpm) }\end{array}$} & \multicolumn{3}{|c|}{ TEMPO (min) } & \multicolumn{3}{|c|}{ RESÍDUO DE MATERIAIS (\%) } & \multirow{3}{*}{ EXTRUSÃO (g) } \\
\hline & \multirow{2}{*}{ A } & \multirow{2}{*}{ B } & \multirow{2}{*}{$\mathbf{T}$} & & TERÇOS & & \\
\hline & & & & CERVICAL & MÉDIO & APICAL & \\
\hline 350 & 4.99 & 7.73 & 12.72 & 59.04 & 69.90 & 60.70 & 0.018 \\
\hline 700 & 2.44 & 6.01 & 8.45 & 86.60 & 78.90 & 66.10 & 0.010 \\
\hline
\end{tabular}

Tabela 2 - Número de acidentes (fraturas de instrumentos, desvios de instrumentação e perfurações) ocorridos durante a desobturação de canais radiculares com instrumentos Quantec SC utilizados nas velocidades de 350 e 700rpm.

\begin{tabular}{|c|c|c|c|}
\hline \multirow{2}{*}{$\begin{array}{l}\text { VELOCIDADE } \\
\text { (rpm) }\end{array}$} & \multicolumn{3}{|l|}{ ACIDENTES } \\
\hline & FRATURA DE INSTRUMENTO & DESVIO & PERFURAÇÃO \\
\hline 350 & $\mathrm{~N}^{0} .1$ (1) $\mathrm{N}^{0} .5$ (3) $\mathrm{N}^{\circ} .6$ (1) $\mathrm{N}^{\circ} .8(0)$ & 4 & 3 \\
\hline 700 & $\mathrm{~N}^{0} .1$ (2) $\mathrm{N}^{0} .5$ (2) $\mathrm{N}^{0} .6(0) \mathrm{N}^{0} .8$ (1) & 2 & 1 \\
\hline
\end{tabular}




\section{Dıscussão}

A total remoção do material obturador das paredes dos canais radiculares é um importante passo no retratamento endodôntico. Esta remoção pode ser feita com instrumentos manuais, oscilatórios ou rotatórios. $1,14,16,17,19,23,24,27$

O instrumento rotatório Quantec SC foi introduzido na endodontia para o tratamento endodôntico tornando-o mais efetivo e em menor tempo. A velocidade recomendada para esse sistema é de $350 \mathrm{rpm}^{4,10}$.

Embora a velocidade recomendada para o retratamento com este sistema seja a de $350 \mathrm{rpm}$, a prática demostrou que a mesma não é a mais adequada pela dificuldade encontrada pelo instrumento em penetrar toda a extensão da obturação.

Mounce ${ }^{17}$ recomendou a velocidade de 900 a 1500rpm para remoção do material obturador com instrumentos rotatórios; Bramante e Betti ${ }^{4}$, recomendaram 700 a $1500 \mathrm{rpm}$.

Neste estudo, o menor tempo para a remoção do material obturador, (Tempo Total), foi encontrado no grupo II (700rpm; $8.58 \mathrm{~min}$ ). A decisão de utilizar as duas velocidades, 350 e $700 \mathrm{rpm}$, baseou-se na orientação do fabricante que recomenda a velocidade de $350 \mathrm{rpm}$, porém, durante a realização deste trabalho, verificou-se dificuldade para trabalhar com essa velocidade, principalmente para atingir a extensão de trabalho. Aumentando a velocidade para 700rpm, a fase de penetração foi facilitada. Como era esperado, com a velocidade de $700 \mathrm{rpm}$ o procedimento de remoção da obturação foi realizado em menor tempo (Tabela 1). Bramante e Betti ${ }^{4}$ encontraram que na velocidade de 1500rpm o sistema Quantec foi mais eficiente em remover obturação com guta-percha, e Schirrmeister et al ${ }^{20,21}$ usaram $350 \mathrm{rpm}$ no sistema RACE para o retratamento.

No presente estudo, resíduos de material obturador de canal foram observados nos dois grupos, entretanto, na velocidade de 700rpm a quantidade de resíduo foi maior (Tabela 2).

A presença de material obturador remanescente tem sido analisada radiograficamente ou por meio de cortes transversais das raízes realizando-se medidas lineares ou atribuindo-se escores ${ }^{2,3,4,5,6,9,10}$ No presente estudo, os dentes foram seccionados longitudinalmente e a quantidade de material residual foi medida com auxilio do computador utilizando o programa Sigma Scan. Este método mostrou, em outros estudos, ser bastante eficiente ${ }^{4,22}$.
Observou-se também, que o instrumento Quantec SC não removeu a obturação em bloco, mas sim em pequenos fragmentos, que, permaneciam aderidos ao instrumento durante muito tempo acompanhando o movimento do mesmo; tal fato pode ser devido ao desenho dos mesmos, o que pode ter determinado a dificuldade em eliminar o material obturador. Durante a ação dos instrumentos na remoção do material obturador, os mesmos são forçados lateralmente contra as paredes do canal, o que oferece uma certa resistência. Por ser de níquel-titânio e apresentar grande módulo de elasticidade o uso do instrumento é mais difícil, principalmente na velocidade de 350rpm onde o atrito com a obturação se faz maior já que a mesma não é plastificada com essa velocidade. A menor quantidade de guta-percha e cimento foi constatada no terço apical, o que pode ser atribuída à forma achatada dos canais dos dentes utilizados, com maior extensão no sentido vestíbulo-lingual a qual torna-se circular à medida que se aproxima do ápice, resultando em melhor contato do instrumento com as paredes do canal. O instrumento permanece centralizado no canal radicular durante o movimento de rotação. Os resíduos encontrados no terço apical eram compostos de gutapercha e cimento, enquanto que nos terços cervical e médio encontrou-se maior quantidade de cimento.

A remoção de obturação durante o retratamento implica em riscos como, perfuração, desvio de instrumentação e fratura de instrumentos ${ }^{122}$. Maior número de fratura de instrumentos foi observado durante a fase de penetração com o instrumento número 5 até a porção apical; o número de instrumentos fraturados foi semelhante nas duas velocidades (Tabela 4). Desvio de instrumentação e perfuração (Tabela 5) foram observados no terço apical, principalmente quando foi utilizada a velocidade de 350rpm, devido à dificuldade encontrada para atingir a extensão de trabalho. Com essa velocidade o atrito do instrumento com a obturação não era suficiente para plastificar a guta-percha, forçando o instrumento contra a parede do canal, determinando o desvio do trajeto original do canal ou a perfuração. Observou-se que o local de fratura do instrumento coincidia com o local da perfuração, o que mostrou que a fase de penetração era a mais complicada causando a fratura do instrumento. Friedman; Rotstein; Shar-Lev ${ }^{8}$ usando instrumento com ponta inativa e solvente encontraram menos acidentes durante o retratamento. Glassman e Serota ${ }^{11}$, Bramante e Betti ${ }^{4}$, recomendam o uso do instrumento Quantec SC combinado com instrumentos manuais. 
Na remoção da obturação, esta combinação é muito importante pois, os instrumentos manuais, criam um caminho para a introdução dos instrumentos Quantec diminuindo, assim, os acidentes tornando o procedimento seguro e com melhor limpeza do canal.

\section{Conclusão}

Todos os canais apresentaram resíduos de material obturador; na velocidade de 700rpm o tempo para a remoção da obturação foi reduzido; quando a velocidade de $350 \mathrm{rpm}$ foi usada, menos resíduos permaneceram no canal; não houve diferença significante quanto à extrusão de material obturador considerando as duas velocidades; fraturas de instrumentos ocorreram em ambas as velocidades e foram mais freqüentes com os instrumentos número 5; desvios de instrumentação e perfurações ocorreram mais na velocidade de 350rpm.

Assim, os instrumentos rotatórios podem ser utilizados na desobturação de canais radiculares, utilizando-se a velocidade de 700rpm.

\begin{abstract}
The aim of this study was compare the ability of Quantec SC rotary system to remove gutta-percha and sealer from root canal when used using speed, 350 or 700rpm. Twelve extracted mandibular incisors were filled by lateral condensation with gutta-percha and Ketac-Endo sealer. After six months the root canal obturations were removed with Quantec SC rotary system, at speed of 350 or $700 \mathrm{rpm}$. The teeth were grooved longitudinally and evaluated using the Sigma Scan II software. Were evaluated: time to gutta-percha removal, canal wall cleanliness and extruded material. The KruskalWallis on-way analysis of variance was used to identify any significance differences amongst two groups. The number of canal transportation and fracture instruments was also recorded. All examined teeth had filling residues in the root canal; in 700rpm the time of work was reduced but at 350rpm more gutta-percha and cements was removed. Extruded material did not show difference at 350 or 700rpm. Procedures errors, instrument fractures occurred in both speed but most frequently in \# 5 instrument; canal transportation and perforation occurred most in 350rpm. According to this study Quantec SC system could be used at $700 \mathrm{rpm}$ to root canal obsturation remotion.
\end{abstract}

\title{
UNITERMS
}

Retreatment; root canal retreatment; Quantec.

\section{REFERENCIAS}

1. Aryanpour S, Van Nieuwenhuysen JP, D'Hoore W. Endodontic retreatment decisions: no consensus. I Endod J.2000 may;33(3):208-18.

2. Basso AL, Silva Neto UX, Westphalen VPD. Análise radiográfica do retratamento endodôntico realizado pela técnica manual, sistema Profile e Protaper. J Bras Endod 2003 abril;4(2):203-7.

3. Bramante CM, Freitas CVJ. Retratamento endodôntico: Estudo comparativo entre técnica manual, ultra-som e Canal Finder. Rev Odon Univ São Paulo 1998 jan/mar;12(1):13-7.

4. Bramante CM, Betti LV. Efficacy of Quantec rotary instruments for gutta-percha removal. I Endod J.2000 sept ;33(5):463-7.

5. Bueno CES, Delboni MG, Araujo RA, Carrara HJ, Cun ha RS. Effectiveness of rotary and hand files in gutta-percha and sealer removal using chloroform or chlorhexidine gel. Braz Dent J 2006 fev.;17(2):139-43.

6. Ferreira JJ, Rhodes JS, Ford TR. The efficacy of gutta-percha removal using ProFile. I Endod J.2001 june;34(4):267-74.

7. Friedman S, Stalbholz A. Endodontic retreatment-case selection and technique. Part 1:criteria for case selection. J Endod 1986 jan;12(1):28-33.

8. Friedman S, Rotstein I, Shar-Lev S. Bypassing gutta-percha root fillings with an automated device. J Endod,1989 sept;15(9):432-7.

9. Friedman S, Moshonov J, Trope M. Efficacy of removing glass ionomer cement, zinc oxide eugenol and epoxy resin sealers from retreatment root canals. Oral Surg.Oral Med.Oral Pathol Oral Radiol.1992 may;73(5):609-12.
10. Gelani V, Hussne RP, Leonardo RT, Capelli A. Remoção do material obturador dos canais radiculares empregando instrumentos de níquel titânio, sistema Quantec, acionados a motor. J Bras Endod 2004 maio;5(17):108-14.

11. Glassman G, Serota K. Endodontics en transition: evolution of the rotary revolution. O Health 1997 oct; 87(10):37-40

12. Hülsmann M, Stotz S. Efficacy, cleaning ability and safety of different devices for gutta-percha removal in root canal retreatment. I Endod J 1997 july ;30(4): 227-33.

13. Imura N, Kato AS, Hata GI, Uemura M, Toda T, Weine F. A comparison of the relative efficacies of four hand and rotary instrumentation techniques during endodontic retreatment. I Endod J. 2000 july;33(4):361-6.

14. -Kvist T, Heden G, Reit C. Endodontic retreatment strategies used by general dental practioners. Oral Surg Oral Med Oral Pathol. Endod 2004 april; $97(4): 502-7$.

15. -Kvist T, Reit T. The perceived benefit of endodontic retreatment. I Endod J. 2002 april;35 (4):359-65.

16. Maciel ACC, Scelza MFZ.Efficacy of automated versus hand instrumentation during root canal retreatment: an ex vivo study.I Endod J 2006 oct;39(10):779-84.

17. Mounce R. Current concepts in gutta-percha removal in endodontic retreatment. NYSDJ 2004 july: 70(7):32-5.

18. Mounce R. Endodontic retreatment possibilities: evaluation, limitations and considerations. Compendium. 2004 may; 25(5):364-8.

19. Saad AY, Al-Hadlag SM, Al-Katheeri NH. Efficacy of two rotary NiTi instruments in the removal of gutta-percha during root canal retreatment. J Endod 2007 jan; 33(1):38-41 
20. Shirrmeister JF, Wrbas KT, Meyer KM, Altenburger MJ, Hellwing E. Efficacy of different rotary instruments for gutta-percha removal in root canal retreatment. J Endod. 2006 may,32(5):469-72.

21. Schirrmeister JF, Meyer KM, Hermanns P, Altenburger MJ, Wrbas KT. Effectiveness of hand and rotary instrumentation for removing a new synthetic polymer-based root canal obturation material (Epiphany) during retreatment. I Endod J. 2006 febrery;39(2):150-6.

22. Souza SMG, Bramante CM. Análise comparativa "in vitro" da desobturação dos canais radiculares utilizando o canal finder associado ao uso de solventes. Rev Bras Odontol, 1994 mar;40(3):2-7.

23. Tanomaru Filho M, Alencar A, Araújo C. Retratamento endodôntico técnica e meios auxiliares. ROBRAC 1996 jun;18(6):25-8.

24. Tanomaru Filho M, Leonardo MR, Silva LAB, Castro ET. Avaliação radiográfica in vitro da capacidade de limpeza de técnicas de retratamento endodôntico Rev APCD.1999 mar; 53(3):238-41.

25. Valois CRA, Navarro M, Ramos AA, Castro AJR, Gahyva SMM. Effectiveness of the Profille 04 taper series 29 files in removal of gutta-percha root fillings during curved root canal retreatment. Braz Dent J 2001dez;12(4):95-9.
26. Zmener O, Pameijer CH, Banegas G. Retreatment efficacy of hand versus automated instrumentation in oval-shaped root canals: an ex vivo study. I Endod J 2006 july;39 (7):521-6.

27. Wilcox L, Van Surksum R. Endodontic retreatment in large and small straight canals. J Endod. 1991 march;17(3):119-21.

Recebido em 22/07/08

Aprovado em 24/02/10

Correspondência:

Clovis Monteiro Bramante

Endereço: Faculdade de Odontologia de Bauru-USP

Al. Octávio Pinheiro Brisola 9-75

CEP: 17012-901

Bauru, SP, Brasil

e-mail: bramante@fob.usp.br 